\title{
Projecting the own affective states onto others: no influence of perceived similarity
}

\author{
Irene Trilla $a^{\mathrm{a}, \mathrm{b}, *}$, Friedrich Eiserbeck ${ }^{\mathrm{a}, \mathrm{b}}$, Isabel Dziobek ${ }^{\mathrm{a}, \mathrm{b}}$ \\ ${ }^{a}$ Berlin School of Mind and Brain, Humboldt-Universität zu Berlin, Berlin, Germany \\ ${ }^{\mathrm{b}}$ Department of Psychology, Institute of Life Sciences, Humboldt-Universität zu Berlin, Berlin, Germany \\ *irene.trilla@hu-berlin.de
}

\begin{abstract}
Research has shown that people project their own emotional experiences when inferring the affective states of others. This preregistered study investigated whether the degree of affective self-projection depends on the perceived similarity with the other, an effect that has been observed for inferences of cognitive mental states. In a within-subject experiment, the perceived similarity between the participants and a series of targets was manipulated using a minimal group paradigm. After being induced positive and negative affective states, participants made emotion judgments of morphed happy and sad facial expressions of a similar and a dissimilar target. Confirming the occurrence of affective self-projection, facial expressions were judged as happier after positive induction than after negative induction. However, contrarily to more cognitive forms of social projection, we did not find evidence to support that the degree of selfprojection depends on the perceived similarity with the other during quick emotion judgments.
\end{abstract}

Keywords: social projection; emotional egocentricity; emotion perception; similarity. 


\section{Introduction}

One common strategy to understand others' mental states is to use one's own thoughts and feelings as a reference, a process referred to as self-projection. Evidence of self-projection comes from studies in which people's beliefs and affective states interfered with their inferences about the experiences of another person (Mitchell, 2009; Samson et al., 2010; Silani et al., 2013; Trilla et al., 2020). This is typically shown by egocentrically-biased judgments about the other's mental and affective states.

Research in social psychology has shown that individuals preferentially rely on their own experiences when making social inferences about people they perceive similar to themselves, such as in-group members (e.g., Ames, 2004b; Clement \& Krueger, 2002; Davis, 2017; Robbins \& Krueger, 2005). To predict the mental states of dissimilar others, people tend to recruit other inferential strategies, such as using implicit beliefs or stereotypes about a particular social group (Ames, 2004b; Ames et al., 2012), or taking as reference a known person who is more similar to the target (Willard \& Markman, 2017). This flexible use of self-projection according to the perceived similarity with the other may guarantee more accurate inferences. Provided that people who share specific characteristics tend to react more alike, attributing their thoughts and feelings onto similar individuals can be an effective inferential strategy, especially when we have little information about the other. Conversely, the use of self-projection to infer the mental states of dissimilar others, or of those who are in a situation incongruent with our own current experience, can lead to stronger egocentric biases (Samson et al., 2010; Silani et al., 2013; Tamir \& Mitchell, 2013). In this case, a process of adjustment is needed to correct away from the self-projection and account for the dissimilarities between oneself and the other (Tamir \& Mitchell, 2013).

So far, the role of perceived similarity has been mainly demonstrated for the projection of cognitive mental states, such as traits, beliefs and attitudes (Ames, 2004b; Ames et al., 2012; Clement \& Krueger, 2002; Davis, 2017). To our knowledge, only one study (O'Brien \& Ellsworth, 2012) has assessed how similarity influences affective self-projection. In this study, participants were deprived of drinking water, or exposed to a cold setting, before inferring the visceral states of a protagonist of a written story. Participants attributed their feelings of coldness and thirst more strongly to protagonists with whom they shared the same political views, compared to protagonists who held opposing attitudes. The study by O_Brien and Ellsworth_(2012) provides initial evidence that perceived similarity influences processes of affective self-projection. However, it is yet to be determined whether such modulation extends to more automatic and low-level forms of emotion inferences. As in the case of cognitive inferences, research has shown that people tend to overattribute their affective states when reading others' overt emotional expressions (Trilla et al., 2020).

To expand our understanding of the role of perceived similarity on affective self-projection, the present study examined the extent to which individuals attribute their affective states when judging emotional facial expressions of similar and dissimilar targets. Similarity was manipulated using a minimal group paradigm that assigned the same or a different arbitrary characteristic to the participant and the evaluated targets. We predicted that emotion attributions would be more biased towards the own affective experiences when judging a similar target compared to a dissimilar target. 


\section{Methods}

\section{Participants}

Sixty-four adults (31 females, 32 males, 1 non-binary; Age: $M=27, S D=5.2$ ) were recruited for this study. The sample size was decided based on a power analysis to detect a small-to-moderate effect size (Cohen's $d=0.35$ ) using a one-tailed paired-samples $t$-test for the main contrast of interest, with alpha $=0.05$ and power $=0.80$ (see preregistration: https://osf.io/kjb84). None of the participants reported current psychiatric or neurological disorders, current psychoactive medication, severe cognitive or neuropsychological impairments, history of regular substance use, or colourblindness.

The study conformed to the Code of Ethics of the World Medical Association (Declaration of Helsinki), and was approved by the Ethics Committee of the Psychology Department at HumboldtUniversität zu Berlin. All participants provided informed written consent.

\section{Materials and procedure}

\section{Similarity manipulation}

A minimal group paradigm based on the one used by Montalan et al. (2012) was conducted to manipulate the similarity between the participant and four target faces. First, participants were asked to estimate the number of dots in 10 stimulus patterns, each presented for $1000 \mathrm{~ms}$. Their estimation performance was ostensibly used to determine the participant's cognitive style (Overestimator or Underestimator). In reality, the assignment of the cognitive style was pre-set and counterbalanced across participants. Participants were informed about their alleged estimation performance, and read a brief description of the two cognitive styles. Overestimators were described as individuals who tend to process numerical information with a stronger focus on quantitative characteristics. Underestimators were described as individuals who tend to process numerical information focusing on qualitative characteristics.

Next, participants were asked to learn the cognitive styles of four different target faces. Two of the targets were assigned the same cognitive style as the participant ("similar targets"), and two had the other cognitive style ("dissimilar targets"). The assignment of the similarity condition to the targets was randomized for each participant. To reduce the influence of non-manipulated factors on the perceived (dis)similarity with the targets, images of four men were used for male participants, and images of four women were used for female participants. The gender of the targets was chosen randomly for participants identified as non-binary. One similar and one dissimilar target would be later presented in the affective self-projection paradigm, and the remaining two targets, in a cognitive self-projection control task.

Participants learnt the targets' cognitive styles through an associative learning task. On each trial, a neutral face of one of the four targets, or the word "You", was presented for $1000 \mathrm{~ms}$. Face stimuli were taken from the FACES database (Ebner 2010; see supplementary material for more details about the stimuli). Next, the response options ("Underestimator", "Overestimator") appeared below the stimulus, on the left and right side of the screen. Participants had to guess the cognitive style of the target by pressing a left or a right keyboard key. In "You" trials, participants had to indicate their own cognitive style. The selected response was highlighted for $500 \mathrm{~ms}$, after which feedback ("Correct" or "False, this person is an [over/under]estimator") appeared below the stimulus for 1000 ms. A blank screen was displayed for $500 \mathrm{~ms}$ before the onset of the next trial. 
Participants completed a minimum of 10 blocks of 5-trials each. In each block, the four target faces, and "You", were presented once in random order. To ensure that participants assimilated the cognitive style of all targets, they completed additional blocks until each stimulus was classified correctly on 8 out of the last 10 times presented, or if a maximum of 30 blocks was reached. On average, participants completed 12.4 blocks $(S D=4.6)$. Data from two participants who did not achieve this learning criterion by the end of the task were excluded from the statistical analyses.

As a manipulation check, participants indicated their perceived similarity with each target before and after the minimal group paradigm using a 9 -point scale $(1=$ Not similar to me; $9=$ Similar to me). Participants also rated how much they liked each target $(1=\mathrm{I}$ don't like this person; $9=\mathrm{I}$ like this person), and how sympathetic they found them $(1=$ Not sympathetic; $9=$ Sympathetic $)$.

\section{Affective self-projection}

A paradigm similar to Trilla et al. (2020) was used to estimate egocentric biases during emotion judgments of a similar and a dissimilar target. In two separate within-subject blocks, participants were first induced positive or negative affective states using audiovisual stimuli. Specifically, participants watched 1-minute videos that displayed a joyful (e.g., playful panda bears) or a sad (e.g., a dog seemingly mourning the death of another dog) animal scene, with instrumental music of matching valence playing in the background. Further details about the affect induction stimuli and procedure are available in the supplementary material. The order of the affect induction (positive affect, negative affect) was counterbalanced across participants. To assess the effectiveness of the affective state manipulations, participants reported their current mood on an 11-point scale $(1=$ negative mood, indicated by a sad emoticon; 11 = positive mood, indicated by a happy emoticon) after watching the videos, and at the end of each block.

Following each affect induction, participants were asked to make quick emotion judgments of facial expressions of a similar and a dissimilar target. Ambiguous emotional expressions were created for each target by mixing an image of a happy face with a sad face at varying degrees (see supplementary material). Each trial of the emotion judgement task began with a fixation cross displayed for $1500 \mathrm{~ms}$. Next, one of the morphed emotional faces of the similar or the dissimilar target was presented for $500 \mathrm{~ms}$. At the stimulus offset, the response options ("happy", "sad") appeared on the right and left side of the screen (location randomly determined for each participant) until the participant made a response via key press.

The morph level presented in each trial was selected based on the participant's previous response following a 1-up/1-down procedure (see supplementary material). This adaptive psychophysical method allowed to reduce the number of trials needed to estimate the participant's point of subjective equality (PSE), which represents the point on a happy-sad morph continuum at which a facial expression is equally likely judged as happy or as sad. Individual PSEs were estimated for the similar and dissimilar targets after each affect induction, and were used as the main dependent variable. According to the morph coding used in this study, lower PSE values were indicative of a higher tendency to evaluate emotional expressions as happy (vs. sad). Based on this, egocentric biases would be shown if PSEs in the positive affect condition were lower compared to the negative affect condition. On average, participants completed 63.4 trials $(S D=9.4)$ per block, which corresponds to approximately 2.6 minutes. 


\section{Cognitive self-projection}

As a control task, we tested self-projection in the cognitive domain using a procedure based on Tamir and Mitchell (2013). This task consisted of three blocks. In the self-block, participants were asked to indicate their attitude towards a series of 30 statements (e.g., "Stormy weather is scary."; see supplementary materials for the complete item list). In the similar- and dissimilar-block, participants had to infer how a similar and a dissimilar target would respond to the same statements. All ratings were made on a 9 -point agreement scale $(1=$ Disagree; $9=$ Agree $)$. A face with a neutral expression was displayed above each statement in the similar- and dissimilar-blocks to indicate the target of the judgment. No reference was made to their cognitive style. Each statement was displayed for $2000 \mathrm{~ms}$ before the appearance of the rating scale, and remained onscreen until the participant responded via mouse click on the rating scale. The order of the statements was randomized. There was no response time limit, but participants were encouraged to answer as quickly as possible without overthinking their response.

To prevent that participants could remember their own ratings, the self-block was completed at the beginning of the experimental session, and the similar- and dissimilar-blocks were conducted after the affective self-projection paradigm, with counterbalanced ordered.

\section{Procedure}

A more detailed description of the complete study procedure is available in the supplementary material. This also includes information about additional measurements conducted at the end of the experimental session that were not used for the current analyses.

\section{Results}

Inferences were based on null hypothesis significance testing with $p<.05$. The Holm-Bonferroni adjustment was used to correct for multiple testing in post-hoc tests following significant interaction effects. Unless prespecified, all statistical tests were two-tailed. Data and R code to reproduce all analyses reported here are available at: https://osf.io/s5gp6/.

\section{Similarity manipulation}

To check whether the minimal group paradigm succeeded in manipulating the participants' perceived similarity with the targets, a repeated-measures analysis of variance (ANOVA) was conducted on the similarity ratings, with targets' Similarity (similar targets, dissimilar targets) and Time (pre-learning, post-learning) as within-subject factors. The ANOVA yielded significant main effects of Similarity, $F(1,61)=13.34, M S E=1.70, p<.001, \eta^{2}{ }_{p}=.179$, and Time, $F(1,61)=15.75$, $M S E=0.80, p<.001, \eta^{2}{ }_{p}=.205$, which were qualified by a significant two-way interaction, $F(1,61)=15.60, M S E=1.53, p<.001, \eta^{2}{ }_{p}=.204$ (supplementary figure S1). Follow-up pairwise comparisons confirmed that, after learning the targets' cognitive styles, those paired with the same cognitive style as the participant were perceived as more similar to themselves $(M=5.19, S D=$ 1.81) than targets with the opposite cognitive style $(M=3.96, S D=1.38), t(61)=4.65, p<.001$, $95 \% \mathrm{CI}=[0.70,1.75]$, Cohen's $d=0.59$. No significant differences in perceived similarity were found between the targets at baseline, $t(61)=-0.09, p=.93,95 \% \mathrm{CI}=[-0.39,0.36]$, Cohen's $d=-$ 0.01 . 
A significant Similarity-by-Time interaction was also found on a repeated-measures ANOVA on likability ratings, $F(1,61)=16.94, M S E=0.94, p<.001, \eta^{2}=.217$, and on sympathy ratings, $F(1,61)=10.80, M S E=0.97, p=.002, \eta^{2}=.150$. Follow-up analyses showed that similar targets were rated as more sympathetic than dissimilar targets after learning their cognitive styles, $t(61)=$ $2.46, p=.05,95 \% \mathrm{CI}=[0.12,1.18]$, Cohen's $d=0.31$, and were also liked more, $t(61)=3.05, p=$ $.01,95 \% \mathrm{CI}=[0.29,1.38]$, Cohen's $d=0.39$. No significant differences on neither sympathy nor likability ratings were found at baseline (all $p>.30$ ). Descriptive statistics for the face ratings are available in the supplementary table S1.

\section{Effects of similarity on affective self-projection}

\section{Affective state manipulation}

A repeated-measures ANOVA on the mood ratings, with Affect induction (positive, negative) and Time of assessment (after affect induction, at the end of the block) as within-subject factors, revealed a significant main effect of affect induction, $F(1,61)=320.00, M S E=5.42, p<.001, \eta^{2}{ }_{\mathrm{p}}$ $=.840$. As expected, participants felt more positively throughout the positive affect block $(M=$ $8.96, S D=1.32)$ than the negative affect block $(M=3.67, S D=1.56)$.

A significant interaction with Time indicated that the strength of the induced mood faded throughout block, $F(1,61)=77.61, M S E=0.61, p<.001, \eta^{2}{ }_{\mathrm{p}}=.560$ (supplementary figure S2). That is, participants reported feeling more positively right after watching the happy video $(M=$ $9.40, S D=1.32)$ than at the end of the block $(M=8.52, S D=1.46), t(61)=7.70, p<.001,95 \%$ $\mathrm{CI}=[0.66,1.12]$, Cohen's $d=0.98$; and more negatively after watching the sad video $(M=3.24$, $S D=1.64)$ than at the end of the block $(M=4.10, S D=1.67), t(61)=-6.21, p<.001,95 \% \mathrm{CI}=$ $[-1.13,-0.58]$, Cohen's $d=-0.79$. Despite the decline of the induced affective state during the emotion judgments, inspection of individual mood ratings confirmed that the emotional videos elicited the expected mood patterns to all participants, so no data had to be excluded on the basis of unsuccessful affect manipulation.

\section{Emotional egocentricity biases}

To check whether the degree of egocentric biases in emotion judgments was modulated by the similarity with the target, we ran a repeated-measures ANOVA on the PSEs, with Affective state (positive, negative) and Similarity (similar target, dissimilar target) as within-subject factors. A significant main effect of Affective state confirmed the occurrence of egocentric biases, $F(1,61)=$ 99.06, $M S E=51.97, p<.001, \eta^{2}{ }_{\mathrm{p}}=.619$. As depicted in a, participants showed a higher tendency to judge the ambiguous facial expressions as happy (indicated by lower PSEs) when they were in a positive affective state $(M=56.73, S D=11.27)$ than when they were in a negative state $(M=$ $65.84, S D=8.85)$.

The predicted interaction with Similarity, however, was not statistically significant, $F(1,61)=0.45$, $M S E=9.85, p=.51, \eta^{2}{ }_{\mathrm{p}}=.007$. This non-significant effect was also shown in the preregistered interaction contrast, which directly compared the bias scores (i.e. the difference in PSE between negative and positive affect conditions) for similar vs. dissimilar targets, $t(61)=0.67, p=.25$ (onetailed), $95 \% \mathrm{CI}=[-0.80, \infty]$, Cohen's $d=0.08$ (Table 1 ). 
Table 1. Mean and standard deviation (SD) of the point of subjective equivalence (PSE) and bias scores estimated for each target and affect block. Positive bias scores reflect egocentric biases.

\begin{tabular}{rllll}
\hline & \multicolumn{2}{c}{ Similar targets } & \multicolumn{2}{c}{ Dissimilar targets } \\
\cline { 2 - 5 } \multicolumn{1}{l}{} & Mean & SD & Mean & SD \\
\hline PSE & & & & \\
Negative affect & 66.14 & 9.93 & 65.55 & 9.94 \\
Positive affect & 56.76 & 12.99 & 56.70 & 11.49 \\
Bias [Neg. - Pos.] & 9.38 & 8.51 & 8.85 & 7.16 \\
\hline
\end{tabular}

To follow-up this null result, we applied equivalence testing on the critical interaction contrast using Two One-Sided Tests (Lakens et al., 2018). This procedure allowed us to test whether egocentric biases were statistically equivalent for similar and dissimilar targets. Equivalence bounds were determined based on the preregistered smallest effect size of interest (Cohen's $d=0.35$ ). A significant equivalence test, $t(61)=-2.09, p=0.02,95 \% \mathrm{CI}=[-0.80,1.86]$, indicated that the egocentric biases when judging emotions of similar targets were statistically equivalent to those of dissimilar targets, thus confirming the null effect of similarity.

In addition, we calculated the Bayes factor to quantify the relative evidence for the null hypothesis (i.e. no differences in bias scores between similar and dissimilar targets) over the alternative hypothesis (i.e. stronger bias scores for similar vs dissimilar targets). Bayesian analysis was performed in JASP (JASP Team, 2020). A Bayesian paired-samples t-test with the default prior set by JASP (i.e. Cauchy distribution centred at 0 with a scale parameter of 0.707 , one-sided) estimated a $\mathrm{BF}_{01}=3.91$. This indicates that, given the data, the null hypothesis was 3.91 times more likely than the alternative hypothesis, which is considered moderate evidence for the null (Lee \& Wagenmakers, 2014).

\section{Effects of similarity on cognitive self-projection}

A linear mixed-effect model (LMM) was used to test if the participant's reported attitudes (selfratings) predicted their inferences about the attitudes of a similar and a dissimilar target (otherratings). The other-ratings were used as dependent variable in the LMM. As fixed effects, we included the Self-ratings (continuous predictor, mean-centred), Similarity (categorical predictor: similar target, dissimilar target), and their interaction. The maximal random effect structure supported by the task design had to be reduced to achieve model convergence and to avoid overparameterization. Principal component analysis was used to detect and remove the random slopes that explained zero variance. The final model included random intercepts for participant, item and target identity, as well as a by-participant random slope for self-ratings and a by-item random slope for similarity. $P$-values were calculated based on Wald-statistics approximation.

The LMM yielded a significant main effect of self-rating, $b=0.11,95 \% \mathrm{CI}=[0.06,0.15], S E=$ $0.02, t=4.60, p<.001$, which was qualified by a significant interaction with similarity, $b=0.26$, $95 \% \mathrm{CI}=[0.21,0.32], S E=0.03, t=9.48, p<.001(\mathrm{~b})$. A simple slopes analysis indicated that, as predicted, self-ratings significantly predicted the judgments about a similar target, $b=0.24,95 \% \mathrm{CI}$ $=[0.19,0.29], S E=0.03, t=8.85, p<.001$. That is, the inferred attitudes for the similar target 
were positively associated with the participant's own ratings about the same statements. In contrast, no significant association between self- and other-ratings was found for dissimilar targets, $b$ $=0.02,95 \% \mathrm{CI}=[-0.03,0.07], S E=0.03, t=0.67, p=.50$.

a

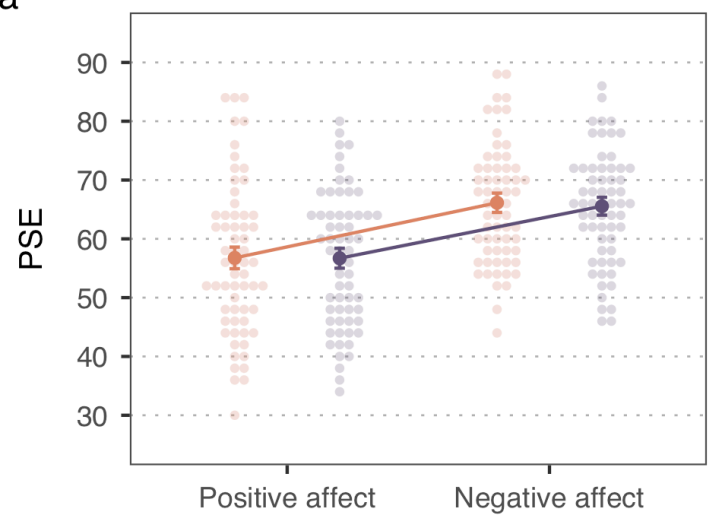

b

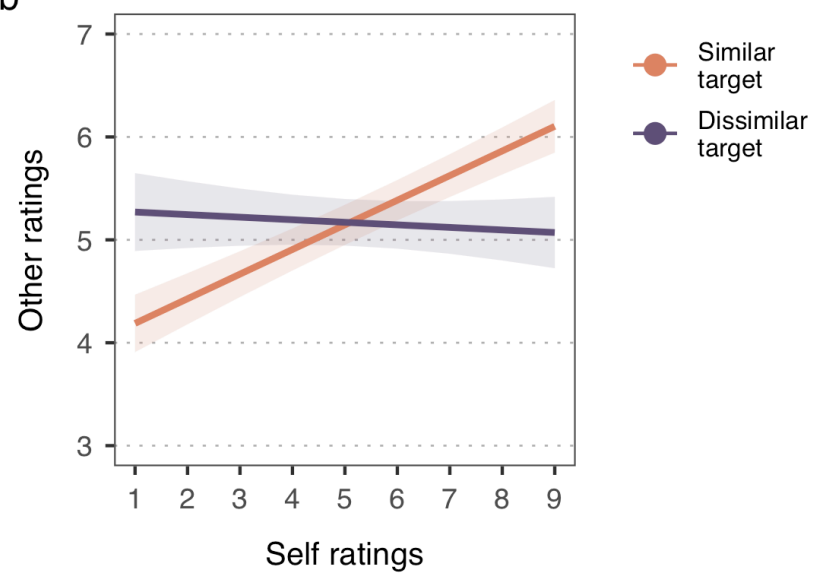

Figure 1. (a) Mean point of subjective equivalence (PSE) estimated for the similar and dissimilar targets in the Emotion judgment task, after positive and negative affect induction. Error bars represent within-subject $95 \%$ confidence intervals. Smaller dots depict the PSE of individual participants. (b) Association between the participant's own attitudes (self ratings) and the attitudes inferred for a similar and a dissimilar target (other ratings) in the cognitive self-projection paradigm, as estimated by the linear mixed model. Shaded areas represent $95 \%$ confidence intervals.

\section{Discussion}

In line with previous studies (e.g.___iedenthal_et_al._2 20000; Trilla et_al. judgments about others' emotional expressions are biased towards the own affective experiences. This supports the idea that people tend to project their own affective states onto others, particularly when only limited information about the target is available (Silani et al., 2013; Trilla et al., 2020; von Mohr et al., 2019). However, contrary to our main prediction, the degree of emotional egocentricity did not significantly differ when judging similar targets compared to dissimilar targets. Our data was more suggestive that similarity does not modulate affective self-projection during quick emotion judgments, at least not to the extent to which it influences cognitive self-projection.

This null effect contrasts with results by O'Brien and Ellsworth (2012), who observed that participants attributed their own visceral states (e.g., thirstiness) more strongly to targets perceived as similar to themselves, compared to dissimilar others. O'Brien and Ellsworth (2012) defined similarity based on shared political attitudes. Thus, it is possible that using a somewhat arbitrary characteristic to manipulate similarity lessened the expected effects in our study. However, this is unlikely as laboratory-induced groups have been shown to elicit stronger effects on social projection than real groups (Robbins \& Krueger, 2005), probably because they avoid confounding influences of pre-existing stereotypes or implicit associations. Moreover, our manipulation significantly impacted the degree of self-projection when making inferences about the targets' attitudes. Replicating previous studies (Ames, 2004b; Ames et al., 2012; Davis, 2017; Tamir \& Mitchell, 2013), participants' self-ratings were predictive of the estimates made about similar targets, but not dissimilar targets. The fact that the ratings for dissimilar targets were not negatively associated with the own attitudes suggests that self-knowledge is not used to predict the reversed attitudes for 
dissimilar targets (Clement \& Krueger, 2002). Instead, previous studies suggest that other types of inferential strategies, such as stereotyping, are recruited when making inferences about dissimilar others (Ames, 2004b, 2004a; Ames et al., 2012; Willard \& Markman, 2017).

Perhaps a more likely explanation of the null result lies in the type of emotion inferences evaluated in this study. So far, evidence of a role of perceived similarity has been found with paradigms in which participants inferred others' mental states based on information about the situation the target is experiencing (Ames, 2004a), or when predicting more stable characteristics such as attitudes and preferences (e.g., Ames, 2004b; Tamir \& Mitchell, 2013). This was also the case in the

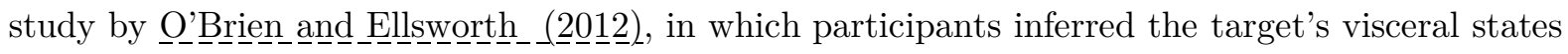
based on the contextual information described in a written story. This type of social inferences requires a cognitive evaluation of the other's situation to predict the other's mental state, a process that may involve perspective-taking. In contrast, attributing affective states based on reading the other's expressive signals is a more automatic and low-level process of social inference, which could be less prone to contextual influences. The short time frame to make the emotion judgments in our task may have also limited the chance to identify the target and recruit the learned information before their inferential process. Future studies should test whether similarity becomes a significant predictor if participants are allowed more time to process the target's identity. Moreover, the role of similarity could be further examined with emotional egocentricity paradigms in which emotion attributions result from perspective-taking processes, rather than an evaluation of the other's

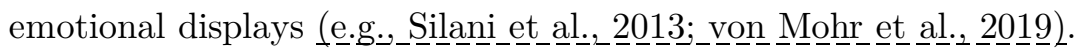

Overall, results from this study strengthen the idea that individuals rely on their own beliefs and emotional experiences to infer what other people think and feel. However, the perceived similarity with the other does not seem to play a significant role in determining the degree of egocentric projections during quick emotion judgments, at least not to the same extent as it influences more evaluative and cognitive forms of social inferences.

\section{Disclosures}

Data, code and online resources: supplementary information, data and code necessary to reproduce all analyses reported here, as well as the preregistration, are available at: osf.io/s5gp6/.

Conflicts of interest: the authors declare no competing interests.

Author contributions: IT and ID conceived the study; IT, FE and ID contributed to the study design; FE collected the data; IT performed the statistical analyses; IT wrote the original draft of the manuscript. All authors contributed to manuscript revision, read and approved the submitted version. 


\section{References}

Ames, D. R. (2004a). Inside the Mind Reader's Tool Kit: Projection and Stereotyping in Mental State Inference. Journal of Personality and Social Psychology, 87(3), 340-353. https://doi.org/10.1037/0022-3514.87.3.340

Ames, D. R. (2004b). Strategies for Social Inference: A Similarity Contingency Model of Projection and Stereotyping in Attribute Prevalence Estimates. Journal of Personality and Social Psychology, 87(5), 573-585. https://doi.org/10.1037/0022-3514.87.5.573

Ames, D. R., Weber, E. U., \& Zou, X. (2012). Mind-reading in strategic interaction: The impact of perceived similarity on projection and stereotyping. Organizational Behavior and Human Decision Processes, 117(1), 96-110. https://doi.org/10.1016/j.obhdp.2011.07.007

Clement, R. W., \& Krueger, J. (2002). Social Categorization Moderates Social Projection. Journal of Experimental Social Psychology, 38(3), 219-231. https://doi.org/10.1006/jesp.2001.1503

Davis, M. H. (2017). Social projection to liked and disliked targets: The role of perceived similarity. Journal of Experimental Social Psychology, 70, 286-293. https://doi.org/10.1016/j.jesp.2016.11.012

JASP Team. (2020). JASP (Version 0.14.1). https://jasp-stats.org/

Lakens, D., Scheel, A. M., \& Isager, P. M. (2018). Equivalence Testing for Psychological Research: A Tutorial: Advances in Methods and Practices in Psychological Science. https://doi.org/10.1177/2515245918770963

Lee, M. D., \& Wagenmakers, E.-J. (2014). Bayesian Cognitive Modeling: A Practical Course. Cambridge University Press.

Mitchell, J. P. (2009). Inferences about mental states. Philosophical Transactions of the Royal Society B: Biological Sciences, 364(1521), 1309-1316. https://doi.org/10.1098/rstb.2008.0318

Montalan, B., Lelard, T., Godefroy, O., \& Mouras, H. (2012). Behavioral Investigation of the Influence of Social Categorization on Empathy for Pain: A Minimal Group Paradigm Study. Frontiers in Psychology, 3. https://doi.org/10.3389/fpsyg.2012.00389

Niedenthal, P. M., Halberstadt, J. B., Margolin, J., \& Innes-Ker, Ä. H. (2000). Emotional state and the detection of change in facial expression of emotion. European Journal of Social Psychology, 30(2), 211-222. https://doi.org/10.1002/(SICI)10990992(200003/04)30:2<211::AID-EJSP988>3.0.CO;2-3

O’Brien, E., \& Ellsworth, P. C. (2012). More Than Skin Deep: Visceral States Are Not Projected Onto Dissimilar Others. Psychological Science, 23(4), 391-396. https://doi.org/10.1177/0956797611432179

Robbins, J. M., \& Krueger, J. I. (2005). Social Projection to Ingroups and Outgroups: A Review and Meta-Analysis. Personality and Social Psychology Review, 9(1), 32-47. https://doi.org/10.1207/s15327957pspr0901_3

Samson, D., Apperly, I. A., Braithwaite, J. J., Andrews, B. J., \& Bodley Scott, S. E. (2010). Seeing it their way: Evidence for rapid and involuntary computation of what other people see. Journal of Experimental Psychology: Human Perception and Performance, 36(5), 12551266. https://doi.org/10.1037/a0018729 
Silani, G., Lamm, C., Ruff, C. C., \& Singer, T. (2013). Right Supramarginal Gyrus Is Crucial to Overcome Emotional Egocentricity Bias in Social Judgments. Journal of Neuroscience, 33(39), 15466-15476. https://doi.org/10.1523/JNEUROSCI.1488-13.2013

Tamir, D. I., \& Mitchell, J. P. (2013). Anchoring and adjustment during social inferences. Journal of Experimental Psychology: General, 142(1), 151-162. https://doi.org/10.1037/a0028232

Trilla, I., Weigand, A., \& Dziobek, I. (2020). Affective states influence emotion perception: Evidence for emotional egocentricity. Psychological Research. https://doi.org/10.1007/s00426-02001314-3

von Mohr, M., Finotti, G., Ambroziak, K. B., \& Tsakiris, M. (2019). Do you hear what I see? An audio-visual paradigm to assess emotional egocentricity bias. Cognition $\mathcal{E}$ Emotion, 1-15. https://doi.org/10.1080/02699931.2019.1683516

Willard, D. F. X., \& Markman, A. B. (2017). Anchoring on Self and Others During Social Inferences. Topics in Cognitive Science, 9(3), 819-841. https://doi.org/10.1111/tops.12275 